\title{
Statistical Summability of Double Sequences through de la Vallée-Poussin Mean in Probabilistic Normed Spaces
}

\author{
S. A. Mohiuddine and Abdullah Alotaibi \\ Department of Mathematics, Faculty of Science, King Abdulaziz University, P.O. Box 80203, Jeddah 21589, Saudi Arabia \\ Correspondence should be addressed to S. A. Mohiuddine; mohiuddine@gmail.com
}

Received 21 September 2013; Accepted 26 October 2013

Academic Editor: Mohammad Mursaleen

Copyright (C) 2013 S. A. Mohiuddine and A. Alotaibi. This is an open access article distributed under the Creative Commons Attribution License, which permits unrestricted use, distribution, and reproduction in any medium, provided the original work is properly cited.

The purpose of this paper is to define some new types of summability methods for double sequences involving the ideas of de la Vallée-Poussin mean in the framework of probabilistic normed spaces and establish some interesting results.

\section{Introduction and Preliminaries}

Throughout the paper, the symbols $\mathbb{N}$ and $\mathbb{R}$ will denote the set of all natural and real numbers, respectively. The notion of convergence for double sequence was introduced by Pringsheim [1]: we say that a double sequence $x=\left(x_{j, k}\right)_{j, k \in \mathbb{N}}$ of reals is convergent to $L$ in Pringsheim's sense (briefly, $(P)$ convergent) provided that given $\epsilon>0$ there exists a positive integer $N$ such that $\left|x_{j, k}-L\right|<\epsilon$ whenever $j, k \geq N$.

The idea of statistical convergence is a generalization of convergence of real sequences which was first presented by Fast [2] and Steinhaus [3], independently. Some of its basic properties and interesting concepts, especially, the notion of statistically Cauchy sequence, were proved by Schoenberg [4], Šalát [5], and Fridy [6]. See, for instance, [7-16] and references therein. Mursaleen and Edely [17] introduced the two-dimensional analogue of natural (or asymptotic) density as follows: let $A \subseteq \mathbb{N} \times \mathbb{N}$ and $A(h, l)=\{j \leq h, k \leq l:(j, k) \in$ $A\}$, where $h, l \in \mathbb{N}$. Then

$$
\begin{aligned}
& \bar{\delta}_{2}(A)=(P) \limsup _{h, l \rightarrow \infty} \frac{|A(h, l)|}{h l}, \\
& \underline{\delta}_{2}(A)=(P) \liminf _{h, l \rightarrow \infty} \frac{|A(h, l)|}{h l}
\end{aligned}
$$

are called the upper and lower asymptotic densities of a twodimensional set $A$, respectively, where the vertical bars stand for cardinality of the enclosed set. If $\bar{\delta}_{2}(A)=\underline{\delta}_{2}(A)$, then

$$
\delta_{2}(A)=(P) \lim _{h, l \rightarrow \infty} \frac{|A(h, l)|}{h l}
$$

is called the double natural density of the set $A$. In the same paper, using the notion of double natural density, they extended the idea of statistical convergence from single to double sequences (for recent work, see [18-23]).

The double sequence $x=\left(x_{j, k}\right)$ is statistically convergent to the number $L$ if, for each $\epsilon>0$, the set $\{(j, k), j \leq h, k \leq l$ : $\left.\left|x_{j, k}-L\right| \geq \epsilon\right\}$ has double natural density zero. We denote this by $S-\lim x=L\left(\right.$ or $\left.x_{j, k} \rightarrow L(S)\right)$.

Mursaleen initiated the notion of $\lambda$-statistical convergence (single sequences) with the help of de la Vallée-Poussin mean, in [24]. For detail of $\lambda$-statistical convergence, one can be referred to [25-31] and many others. In [32], Mursaleen et al. presented the notion of $(\lambda, \mu)$-statistical convergence and $(\lambda, \mu)$-statistically bounded for double sequences and showed that $(\lambda, \mu)$-statistically bounded double sequences are $(\lambda, \mu)$-statistical convergence if and only if $(\lambda, \mu)$-statistical limit infimum of $x=\left(x_{j, k}\right)$ is equal to $(\lambda, \mu)$-statistical limit supremum of $x$ (also see [33]).

Suppose that $\lambda=\left(\lambda_{m}\right)$ and $\mu=\left(\mu_{n}\right)$ are two nondecreasing sequences of positive real numbers such that

$$
\begin{gathered}
\lambda_{m+1} \leq \lambda_{m}+1, \quad \lambda_{1}=0, \\
\mu_{n+1} \leq \mu_{n}+1, \quad \mu_{1}=0
\end{gathered}
$$

and each tends to infinity. 
Recall that $(\lambda, \mu)$-density of the set $K \subseteq \mathbb{N} \times \mathbb{N}$ is given by

$$
\begin{aligned}
\delta_{\lambda, \mu}(K)= & (P) \lim _{m, n} \frac{1}{\lambda_{m} \mu_{n}} \\
& \times \mid\left\{m-\lambda_{m}+1 \leq j \leq m,\right. \\
& \left.n-\mu_{n}+1 \leq k \leq n:(j, k) \in K\right\} \mid
\end{aligned}
$$

provided that the limit exists.

We remark, that, for $\lambda_{m}=m$ and $\mu_{n}=n$, the above density reduces to the double natural density.

The generalized double de la Vallée-Poussin mean is defined as

$$
t_{m, n}(x)=\frac{1}{\lambda_{m} \mu_{n}} \sum_{j \in J_{m}} \sum_{k \in I_{n}} x_{j, k}
$$

where $J_{m}=\left[m-\lambda_{m}+1, m\right]$ and $I_{n}=\left[n-\mu_{n}+1, n\right]$.

We say that $x=\left(x_{j, k}\right)$ is $(\lambda, \mu)$-statistically convergent to the number $L$ if, for every $\epsilon>0$,

$$
\text { (P) } \lim _{m, n} \frac{1}{\lambda_{m} \mu_{n}}\left|\left\{j \in J_{m}, k \in I_{n}:\left|x_{j, k}-L\right| \geq \epsilon\right\}\right|=0 \text {. }
$$

We denote this by $S_{\lambda, \mu}-\lim x=L$.

The symbol $\Delta^{+}$will denote the set of all distribution functions (d.f.) $f: \mathbb{R} \rightarrow[0,1]$ which are nondecreasing, left continuous on $\mathbb{R}$, equal to zero on $[-\infty, 0]$, and such that $f(+\infty)=1$. The space $\Delta^{+}$is partially ordered by the usual pointwise ordering of functions.

A triangular norm (or a $t$-norm) [34] is a binary operation $\tau:[0,1] \times[0,1] \rightarrow[0,1]$ which satisfies the following conditions. For all $h_{1}, h_{2}, h_{3} \in[0,1]$

(i) $\tau\left(\tau\left(h_{1}, h_{2}\right), h_{3}\right)=\tau\left(h_{1}, \tau\left(h_{2}, h_{3}\right)\right)$,

(ii) $\tau\left(h_{1}, h_{2}\right)=\tau\left(h_{2}, h_{1}\right)$,

(iii) $\tau\left(h_{1}, h_{3}\right) \leq \tau\left(h_{2}, h_{3}\right)$ whenever $h_{1} \leq h_{2}$,

(iv) $\tau\left(h_{1}, 1\right)=h_{1}$.

In the literature, we have two definitions of probabilistic normed space or, briefly, $\mathrm{PN}$-space; the original one is given by Šerstnev [35] in 1962 who used the concept of Menger [36] to define such space and the other one by Alsina et al. [37] (for more details, see [38-40]).

According to Šerstnev [35], a probabilistic normed space is a triple $(X, v, \tau)$, where $X$ is a real linear space, $v$ is the probabilistic norm, that is, $v$ is a function from $X$ into $\Delta^{+}$, for $x \in X$, the d.f. $v(x)$ is denoted by $\nu_{x}, v_{x}(t)$ which is the value of $v_{x}$ at $t \in \mathbb{R}$, and $\tau$ is a $t$-norm that satisfies the following conditions:

(i) $v_{x}(0)=0$;

(ii) $v_{x}(t)=1$ for all $t>0$ if and only if $x=0$;

(iii) $\nu_{\alpha x}(t)=v_{x}(t /|\alpha|)$ for all $t>0, \alpha \in \mathbb{R}$ with $\alpha \neq 0$ and $x \in X$;

(iv) $v_{x+y}\left(t_{1}+t_{2}\right) \geq \tau\left(v_{x}\left(t_{1}\right), v_{y}\left(t_{2}\right)\right)$ for all $x, y \in X$ and $t_{1}, t_{2} \in \mathbb{R}^{+}=\{x \in \mathbb{R}: x \geq 0\}$.

\section{Main Results}

We define the notions of $(\lambda, \mu)$-summable, statistically $(\lambda, \mu)$ summable, statistically $(\lambda, \mu)$-Cauchy, and statistically $(\lambda, \mu)$ complete for double sequences with respect to $\mathrm{PN}$-space and establish some interesting results.

Definition 1. A double sequence $x=\left(x_{j, k}\right)$ is said to be $(\lambda, \mu)$ summable in $(X, \nu, \tau)$ (or, shortly, $\nu(\lambda, \mu)$-summable) to $L$ if for each $\epsilon>0, \theta \in(0,1)$ there exists $N \in \mathbb{N}$ such that $v_{t_{m, n}(x)-L}(\epsilon)>1-\theta$ for all $m, n \geq N$. In this case, one writes $\nu(\lambda, \mu)-\lim x=L$.

Definition 2. A double sequence $x=\left(x_{j, k}\right)$ is said to be statistically $(\lambda, \mu)$-summable in $(X, \nu, \tau)$ (or, shortly, $\nu\left(S_{\lambda, \mu}\right)$ summable) to $L$ if $\delta_{2}\left(K_{\lambda, \mu}\right)=0$, where $K_{\lambda, \mu}=\{(m, n) \epsilon$ $\left.\mathbb{N} \times \mathbb{N}: v_{t_{m, n}(x)-L}(\epsilon) \leq 1-\theta\right\}$; that is, if, for each $\epsilon>0$, $\theta \in(0,1)$

$$
(P) \lim _{h, l} \frac{1}{h l}\left|\left\{m \leq h, n \leq l: v_{t_{m, n}(x)-L}(\epsilon) \leq 1-\theta\right\}\right|=0
$$

or equivalently

$$
\text { (P) } \lim _{h, l} \frac{1}{h l}\left|\left\{m \leq h, n \leq l: v_{t_{m, n}(x)-L}(\epsilon)>1-\theta\right\}\right|=1 .
$$

In this case, we write $\nu\left(S_{\lambda, \mu}\right)$-lim $x=L$, and $L$ is called the $\nu\left(S_{\lambda, \mu}\right)$-limit of $x$.

Definition 3. A double sequence $x=\left(x_{j, k}\right)$ is said to be statistically $(\lambda, \mu)$-Cauchy in $(X, \nu, \tau)$ (or, shortly, $\nu\left(S_{\lambda, \mu}\right)$ Cauchy) if, for every $\epsilon>0$ and $\theta \in(0,1)$, there exist $M, N \in \mathbb{N}$ such that, for all $m, p \geq M, n, q \geq M$, the set $S_{\epsilon}(\lambda, \mu)=\left\{(m, n) \in \mathbb{N} \times \mathbb{N}: v_{t_{m, n}(x)-t_{p, q}(x)}(\epsilon) \leq 1-\theta\right\}$ has double natural density zero; that is,

$$
\text { (P) } \lim _{h, l} \frac{1}{h l}\left|\left\{m \leq h, n \leq l: v_{t_{m, n}(x)-t_{p, q}(x)}(\epsilon) \leq 1-\theta\right\}\right|=0 .
$$

Theorem 4. If a double sequence $x=\left(x_{j, k}\right)$ is statistically $(\lambda, \mu)$-summable in $(X, \nu, \tau)$, that is, $\nu\left(S_{\lambda, \mu}\right)$-lim $x=L$ exists, then $\nu\left(S_{\lambda, \mu}\right)$-limit of $\left(x_{j, k}\right)$ is unique.

Proof. Assume that $\nu\left(S_{\lambda, \mu}\right)-\lim x=L_{1}$ and $\nu\left(S_{\lambda, \mu}\right)-\lim x=$ $L_{2}$. We have to prove that $L_{1} \neq L_{2}$. For given $\epsilon>0$, choose $q>0$ such that

$$
\tau((1-q),(1-q))>1-\epsilon .
$$

Then, for any $t>0$, we define

$$
\begin{aligned}
& M_{q}^{\prime}(\lambda, \mu)=\left\{(m, n) \in \mathbb{N} \times \mathbb{N}: v_{t_{m, n}(x)-L_{1}}(t) \leq 1-q\right\}, \\
& M_{q}^{\prime \prime}(\lambda, \mu)=\left\{(m, n) \in \mathbb{N} \times \mathbb{N}: v_{t_{m, n}}(x)-L_{2}(t) \leq 1-q\right\} .
\end{aligned}
$$

Since $\nu\left(S_{\lambda, \mu}\right)$-lim $x=L_{1}$ implies $\delta_{2}\left(M_{q}^{\prime}(\lambda, \mu)\right)=0$ and similarly we have $\delta_{2}\left(M_{q}^{\prime \prime}(\lambda, \mu)\right)=0$. Now, let $M_{q}(\lambda, \mu)=M_{q}^{\prime}$ $(\lambda, \mu) \cap M_{q}^{\prime \prime}(\lambda, \mu)$. It follows that $\delta_{2}\left(M_{q}(\lambda, \mu)\right)=0$ and hence 
the complement $M_{q}^{c}(\lambda, \mu)$ is nonempty set and $\delta_{2}\left(M_{q}^{c}(\lambda, \mu)\right)=$ 1. Now, if $(m, n) \in \mathbb{N} \times \mathbb{N} \backslash M_{q}(\lambda, \mu)$, then

$$
\begin{aligned}
v_{L_{1}-L_{2}}(t) & \geq \tau\left(v_{t_{m, n}(x)-L_{1}}\left(\frac{t}{2}\right), v_{t_{m, n}(x)-L_{2}}\left(\frac{t}{2}\right)\right) \\
& >\tau((1-q),(1-q))>1-\epsilon .
\end{aligned}
$$

Since $\epsilon>0$ was arbitrary, we obtain $\nu_{L_{1}-L_{2}}(t)=1$ for all $t>0$. Hence $L_{1}=L_{2}$. This means that $\nu\left(S_{\lambda, \mu}\right)$-limit is unique.

Theorem 5. If a double sequence $x=\left(x_{j, k}\right)$ is $\nu(\lambda, \mu)$ summable to $L$, then it is $\nu\left(S_{\lambda, \mu}\right)$-summable to the same limit.

Proof. Let us consider that $\nu(\lambda, \mu)-\lim x=L$. For every $\epsilon>0$ and $t>0$, there exists a positive integer $N$ such that

$$
v_{t_{m, n}(x)-L}(t)>1-\epsilon
$$

holds for all $m, n \geq N$. Since

$$
K_{\epsilon}(\lambda, \mu):=\left\{(m, n) \in \mathbb{N} \times \mathbb{N}: v_{t_{m, n}(x)-L}(t) \leq 1-\epsilon\right\}
$$

is contained in $\mathbb{N} \times \mathbb{N}$, hence $\delta_{2}\left(K_{\epsilon}(\lambda, \mu)\right)=0$; that is, $x=$ $\left(x_{j, k}\right)$ is $\nu\left(S_{\lambda, \mu}\right)$-summable to $L$.

Example 6. This example proves that the converse of Theorem 5 need not be true. We denote by $(\mathbb{R},|\cdot|)$ the set of all real numbers with the usual norm and $\tau(a, b)=a b$ for all $a, b \in[0,1]$. Assume that $\nu_{x}(t)=t /(t+|x|)$ for all $x \in X$ and all $t>0$. Here, we observe that $(\mathbb{R}, \nu, \tau)$ is a PN-space. The double sequence $x=\left(x_{j, k}\right)$ is defined by

$$
t_{m, n}(x)= \begin{cases}m n ; & \text { if } m, n=w^{2}, w \in \mathbb{N} \\ 0 ; & \text { otherwise }\end{cases}
$$

For $\epsilon>0$ and $t>0$, write

$$
K_{\epsilon}(\lambda, \mu)=\left\{(m, n) \in \mathbb{N} \times \mathbb{N}: v_{t_{m, n}(x)}(t) \leq 1-\epsilon\right\} .
$$

It is easy to see that

$$
\begin{aligned}
v_{t_{m, n}(x)}(t) & =\frac{t}{t+\left|t_{m, n}(x)\right|} \\
& = \begin{cases}\frac{t}{t+m n}, & \text { for } m, n=w^{2}, w \in \mathbb{N} ; \\
1, & \text { otherwise; }\end{cases}
\end{aligned}
$$

and hence

$$
\lim v_{t_{m, n}(x)}(t)= \begin{cases}0, & \text { for if } m, n=w^{2}, w \in \mathbb{N} \\ 1, & \text { otherwise. }\end{cases}
$$

We see that the sequence $\left(x_{j, k}\right)$ is not $(\lambda, \mu)$-summable in $(\mathbb{R}, \nu, \tau)$. But the set $K_{\epsilon}(\lambda, \mu)$ has double natural density zero since $K_{\epsilon}(\lambda, \mu) \subset\{(1,1),(4,4),(9,9),(16,16), \ldots\}$. From here, we conclude that the converse of Theorem 5 need not be true.

Theorem 7. A double sequence $x=\left(x_{j, k}\right)$ is $\nu\left(S_{\lambda, \mu}\right)$-summable to $L$ if and only if there exists a subset $K=\left\{\left(j_{m}, k_{n}\right): j_{1}<j_{2}<\right.$ $\left.\cdots<j_{m}<\cdots ; k_{1}<k_{2}<\cdots<k_{n}<\cdots\right\} \subseteq \mathbb{N} \times \mathbb{N}$ such that $\delta_{2}(K)=1$ and $\nu(\lambda, \mu)-\lim x_{j_{m}, k_{n}}=L$.
Proof. Assume that there exists a subset $K=\left\{\left(j_{m}, k_{n}\right): j_{1}<\right.$ $\left.j_{2}<\cdots<j_{m}<\cdots ; k_{1}<k_{2}<\cdots<k_{n}<\cdots\right\} \subseteq \mathbb{N} \times \mathbb{N}$ such that $\delta_{2}(K)=1$ and $\nu(\lambda, \mu)-\lim x_{j_{m}, k_{n}}=L$. Then there exists $N \in \mathbb{N}$ such that

$$
v_{t_{m, n}(x)-L}(t)>1-\epsilon
$$

holds for all $m, n>N$. Put $K_{\epsilon}(\lambda, \mu)=\{(m, n) \in \mathbb{N} \times \mathbb{N}$ : $\left.v_{t_{j_{m}, k_{n}}(x)-\xi}(t) \leq 1-\epsilon\right\}$ and $K^{\prime}=\left\{\left(j_{N+1}, k_{N+1}\right),\left(j_{N+2}, k_{N+2}\right), \ldots\right\}$. Then $\delta_{2}\left(K^{\prime}\right)=1$ and $K_{\epsilon}(\lambda, \mu) \subseteq \mathbb{N}-K^{\prime}$ which implies that $\delta_{2}\left(K_{\epsilon}(\lambda, \mu)\right)=0$. Hence $x=\left(x_{j, k}\right)$ is statistically $(\lambda, \mu)$ summable to $L$ in $\mathrm{PN}$-space.

Conversely, suppose that $x=\left(x_{j, k}\right)$ is $\nu\left(S_{\lambda, \mu}\right)$-summable to $L$. For $q=1,2,3, \ldots$ and $t>0$, write

$$
\begin{gathered}
K_{q}(\lambda, \mu)=\left\{(m, n) \in \mathbb{N} \times \mathbb{N}: v_{t_{j_{m}, k_{n}}(x)-L}(t) \leq 1-\frac{1}{q}\right\}, \\
M_{q}(\lambda, \mu)=\left\{(m, n) \in \mathbb{N} \times \mathbb{N}: v_{t_{j_{m}, k_{n}}(x)-L}(t)>\frac{1}{q}\right\} .
\end{gathered}
$$

Then $\delta_{2}\left(K_{q}(\lambda, \mu)\right)=0$ and

$$
\begin{gathered}
M_{1}(\lambda, \mu) \supset M_{2}(\lambda, \mu) \supset \cdots M_{i}(\lambda, \mu) \supset M_{i+1}(\lambda, \mu) \supset \cdots, \\
\delta_{2}\left(M_{q}(\lambda, \mu)\right)=1, \quad q=1,2, \cdots .
\end{gathered}
$$

Now, we have to show that, for $(m, n) \in M_{q}(\lambda, \mu), x=$ $\left(x_{j_{m}, k_{n}}\right)$ is $\nu(\lambda, \mu)$-summable to $L$. Suppose that $x=\left(x_{j_{m}, k_{n}}\right)$ is not $\nu(\lambda, \mu)$-summable to $L$. Therefore, there is $\epsilon>0$ such that $v_{t_{j_{m}, k_{n}}-L}(t) \leq \epsilon$ for infinitely many terms. Let

$$
M_{\epsilon}(\lambda, \mu)=\left\{(m, n) \in \mathbb{N} \times \mathbb{N}: v_{t_{j_{m}, k_{n}}-\xi}(t)>\epsilon\right\}
$$

and $\epsilon>1 / q$ with $q=1,2,3, \ldots$ Then

$$
\delta\left(M_{\epsilon}(\lambda, \mu)\right)=0,
$$

and by $(21), M_{q}(\lambda, \mu) \subset M_{\epsilon}(\lambda, \mu)$. Hence $\delta\left(M_{q}(\lambda, \mu)\right)=0$, which contradicts $(22)$ and therefore $x=\left(x_{j_{m}, k_{n}}\right)$ is $\nu(\lambda, \mu)$ summable to $L$.

Theorem 8. If a double sequence $x=\left(x_{j, k}\right)$ is statistically $(\lambda, \mu)$-summable in PN-space, then it is statistically $(\lambda, \mu)$ Cauchy.

Proof. Suppose that $\nu\left(S_{\lambda, \mu}\right)-\lim x=L$. Let $\epsilon>0$ be a given number so that we choose $q>0$ such that

$$
\tau((1-q),(1-q))>1-\epsilon .
$$

Then, for $t>0$, we have

$$
\delta_{2}\left(A_{q}(\lambda, \mu)\right)=0
$$


where $A_{q}(\lambda, \mu)=\left\{(m, n) \in \mathbb{N} \times \mathbb{N}: v_{t_{m, n}(x)-L}(t / 2) \leq 1-q\right\}$ which implies that

$$
\begin{aligned}
& \delta_{2}\left(A_{q}^{c}(\lambda, \mu)\right) \\
& \quad=\delta_{2}\left(\left\{(m, n) \in \mathbb{N} \times \mathbb{N}: v_{t_{m, n}(x)-L}\left(\frac{t}{2}\right)>1-q\right\}\right) \\
& \quad=1 .
\end{aligned}
$$

Let $(f, g) \in A_{q}^{c}(\lambda, \mu)$. Then $v_{t_{f, g}(x)-L}(t / 2)>1-q$.

Now, let

$$
B_{\epsilon}(\lambda, \mu)=\left\{(m, n) \in \mathbb{N} \times \mathbb{N}: v_{t_{m, n}(x)-t_{f, g}(x)}(t) \leq 1-\epsilon\right\} .
$$

We need to show that $B_{\epsilon}(\lambda, \mu) \subset A_{q}(\lambda, \mu)$. Let $(m, n) \in B_{\epsilon}$ $(\lambda, \mu) \backslash A_{q}(\lambda, \mu)$. Then $v_{t_{m, n}(x)-t_{f, g}(x)}(t) \leq 1-\epsilon, \quad v_{t_{m, n}(x)-L}(t / 2)>$ $1-q$, and in particular $v_{t_{f, g}(x)-L}(t / 2)>1-q$. Then

$$
\begin{aligned}
1-\epsilon & \geq v_{t_{m, n}(x)-t_{f, g}(x)}(t) \\
& \geq \tau\left(v_{t_{m, n}(x)-L}\left(\frac{t}{2}\right), v_{t_{f, g}(x)-L}\left(\frac{t}{2}\right)\right) \\
& >\tau((1-q),(1-q))>1-\epsilon,
\end{aligned}
$$

which is not possible. Hence $B_{\epsilon}(\lambda, \mu) \subset A_{q}(\lambda, \mu)$. Therefore, by $(26) \delta_{2}\left(B_{\epsilon}(\lambda, \mu)\right)=0$. Hence, $x$ is statistically $(\lambda, \mu)$ Cauchy in $\mathrm{PN}$-space.

Definition 9. Let $(X, v, \tau)$ be a PN-space. Then,

(i) PN-space is said to be complete if every Cauchy double sequence is $P$-convergent in $(X, \nu, \tau)$;

(ii) $\mathrm{PN}$-space is said to be statistically $(\lambda, \mu)$-complete (or, shortly, $\nu\left(S_{\lambda, \mu}\right)$-complete) if every statistically $(\lambda, \mu)$-Cauchy sequence in $\mathrm{PN}$-space is statistically $(\lambda, \mu)$-summable.

Theorem 10. Every probabilistic normed space $(X, \nu, \tau)$ is $\nu\left(S_{\lambda, \mu}\right)$-complete but not complete in general.

Proof. Suppose that $x=\left(x_{j, k}\right)$ is $\nu\left(S_{\lambda, \mu}\right)$-Cauchy but not $\nu\left(S_{\lambda, \mu}\right)$-summable. Then there exist $M, N \in \mathbb{N}$ such that, for all $m, p \geq M, n, q \geq M$, the set $E_{\epsilon}(\lambda, \mu)=\{(m, n) \in \mathbb{N} \times \mathbb{N}$ : $\left.v_{t_{m, n}(x)-t_{p, q}(x)}(t) \leq 1-\epsilon\right\}$ has double natural density zero; that is, $\delta_{2}\left(E_{\epsilon}(\lambda, \mu)\right)=0$ and

$$
\begin{aligned}
\delta_{2}\left(F_{\epsilon}\right. & (\lambda, \mu)) \\
& =\delta_{2}\left(\left\{(m, n) \in \mathbb{N} \times \mathbb{N}: v_{t_{m, n}(x)-L}\left(\frac{t}{2}\right)>1-\epsilon\right\}\right) \\
& =0 .
\end{aligned}
$$

This implies that $\delta_{2}\left(F_{\epsilon}^{c}(\lambda, \mu)\right)=1$, since

$$
v_{t_{m, n}(x)-t_{p, q}(x)}(t) \geq 2 v_{t_{m, n}(x)-L}\left(\frac{t}{2}\right)>1-\epsilon,
$$

if $v_{t_{m, n}(x)-L}(t / 2)>(1-\epsilon) / 2$. Therefore $\delta_{2}\left(E_{\epsilon}^{c}(\lambda, \mu)\right)=0$; that is, $\delta_{2}\left(E_{\epsilon}(\lambda, \mu)\right)=1$, which leads to a contradiction, since $x=$ $\left(x_{j, k}\right)$ was $\nu\left(S_{\lambda, \mu}\right)$-Cauchy. Hence $x=\left(x_{j, k}\right)$ must be $\nu\left(S_{\lambda, \mu}\right)$ summable.

To see that a probabilistic normed space is not complete in general, we have the following example.

Example 11. Let $X=(0,1]$ and $\nu_{x}(t)=t /(t+|x|)$ for $t>0$. Then $(X, v, \tau)$ is a probabilistic normed space but not complete, since the double sequence $(1 / \mathrm{mn})$ is Cauchy with respect to $(X, \nu, \tau)$ but not $P$-convergent with respect to the present $\mathrm{PN}$-space.

\section{Conflict of Interests}

The authors declare that there is no conflict of interests regarding the publication of this paper.

\section{Acknowledgments}

This project was funded by the Deanship of Scientific Research (DSR), King Abdulaziz University, Jeddah, under Grant no. (303/130/1433). The authors, therefore, acknowledge with thanks DSR technical and financial support.

\section{References}

[1] A. Pringsheim, "Zur theorie der zweifach unendlichen zahlenfolgen," Mathematische Annalen, vol. 53, no. 3, pp. 289-321, 1900.

[2] H. Fast, "Sur la convergence statistique," Colloquium Mathematicum, vol. 2, pp. 241-244, 1951.

[3] H. Steinhaus, "Sur la convergence ordinaire et la convergence asymptotique," Colloquium Mathematicum, vol. 2, pp. 73-74, 1951.

[4] I. J. Schoenberg, "The integrability of certain function and related summability methods," The American Mathematical Monthly, vol. 66, pp. 361-375, 1959.

[5] T. Šalát, "On statistically convergent sequences of real numbers," Mathematica Slovaca, vol. 30, no. 2, pp. 139-150, 1980.

[6] J. A. Fridy, "On statistical convergence," Analysis, vol. 5, no. 4, pp. 301-313, 1985.

[7] H. Çakalli and M. K. Khan, "Summability in topological spaces," Applied Mathematics Letters, vol. 24, no. 3, pp. 348-352, 2011.

[8] B. Hazarika, "On generalized statistical convergence in random 2-normed spaces," Scientia Magna, vol. 8, no. 1, pp. 58-67, 2012.

[9] S. A. Mohiuddine and M. A. Alghamdi, "Statistical summability through a lacunary sequence in locally solid Riesz spaces," Journal of Inequalities and Applications, vol. 2012, article 225, 2012.

[10] S. A. Mohiuddine and M. Aiyub, "Lacunary statistical convergence in random 2-normed spaces," Applied Mathematics \& Information Sciences, vol. 6, no. 3, pp. 581-585, 2012.

[11] S. A. Mohiuddine, H. Şevli, and M. Cancan, "Statistical convergence in fuzzy 2-normed space," Journal of Computational Analysis and Applications, vol. 12, no. 4, pp. 787-798, 2010.

[12] M. Mursaleen, "On statistical convergence in random 2-normed spaces," Acta Scientiarum Mathematicarum, vol. 76, no. 1-2, pp. 101-109, 2010. 
[13] M. Mursaleen and O. H. H. Edely, "Generalized statistical convergence," Information Sciences, vol. 162, no. 3-4, pp. 287294, 2004.

[14] M. Mursaleen, V. Karakaya, M. Ertürk, and F. Gürsoy, "Weighted statistical convergence and its application to Korovkin type approximation theorem," Applied Mathematics and Computation, vol. 218, no. 18, pp. 9132-9137, 2012.

[15] A. Şahiner, M. Gürdal, S. Saltan, and H. Gunawan, "Ideal convergence in 2-normed spaces," Taiwanese Journal of Mathematics, vol. 11, no. 5, pp. 1477-1484, 2007.

[16] U. Yamancı and M. Gürdal, "On lacunary ideal convergence in random 2-normed space," Journal of Mathematics, vol. 2013, Article ID 868457, 8 pages, 2013.

[17] M. Mursaleen and O. H. H. Edely, "Statistical convergence of double sequences," Journal of Mathematical Analysis and Applications, vol. 288, no. 1, pp. 223-231, 2003.

[18] M. Mursaleen and S. A. Mohiuddine, "Statistical convergence of double sequences in intuitionistic fuzzy normed spaces," Chaos, Solitons \& Fractals, vol. 41, no. 5, pp. 2414-2421, 2009.

[19] S. A. Mohiuddine, H. Şevli, and M. Cancan, "Statistical convergence of double sequences in fuzzy normed spaces," Filomat, vol. 26, no. 4, pp. 673-681, 2012.

[20] R. Çolak and Y. Altin, "Statistical convergence of double sequences of order $\widetilde{\alpha}$, Journal of Function Spaces and Applications, vol. 2013, Article ID 682823, 5 pages, 2013.

[21] S. A. Mohiuddine, A. Alotaibi, and M. Mursaleen, "Statistical convergence of double sequences in locally solid Riesz spaces," Abstract and Applied Analysis, vol. 2012, Article ID 719729, 9 pages, 2012.

[22] S. A. Mohiuddine, B. Hazarika, and A. Alotaibi, "Double lacunary density and some inclusion results in locally solid Riesz spaces," Abstract and Applied Analysis, vol. 2013, Article ID 507962, 8 pages, 2013.

[23] H. Dutta, "A characterization of the class of statistically preCauchy double sequences of fuzzy numbers," Applied Mathematics \& Information Sciences, vol. 7, no. 4, pp. 1437-1440, 2013.

[24] M. Mursaleen, " $\lambda$-statistical convergence," Mathematica Slovaca, vol. 50, no. 1, pp. 111-115, 2000

[25] C. Belen and S. A. Mohiuddine, "Generalized weighted statistical convergence and application," Applied Mathematics and Computation, vol. 219, no. 18, pp. 9821-9826, 2013.

[26] R. Çolak and C. A. Bektaş, " $\lambda$-statistical convergence of order $\alpha$," Acta Mathematica Scientia B, vol. 31, no. 3, pp. 953-959, 2011.

[27] H. Dutta and T. Bilgin, "Strongly $\left(V^{\lambda}, A, \Delta_{v m}^{n}, p\right)$-summable sequence spaces defined by an Orlicz function," Applied Mathematics Letters, vol. 24, no. 7, pp. 1057-1062, 2011.

[28] O. H. H. Edely, S. A. Mohiuddine, and A. K. Noman, "Korovkin type approximation theorems obtained through generalized statistical convergence," Applied Mathematics Letters, vol. 23, no. 11, pp. 1382-1387, 2010.

[29] M. Et, M. Çınar, and M. Karakaş, "On $\lambda$-statistical convergence of order $\alpha$ of sequences of function," Journal of Inequalities and Applications, vol. 2013, article 204, 2013.

[30] S. A. Mohiuddine and Q. M. D. Lohani, "On generalized statistical convergence in intuitionistic fuzzy normed space," Chaos, Solitons \& Fractals, vol. 42, no. 3, pp. 1731-1737, 2009.

[31] S. A. Mohiuddine, A. Alotaibi, and M. Mursaleen, "A new variant of statistical convergence," Journal of Inequalities and Applications, vol. 2013, article 309, 2013.
[32] M. Mursaleen, C. Çakan, S. A. Mohiuddine, and E. Savaş, "Generalized statistical convergence and statistical core of double sequences," Acta Mathematica Sinica, vol. 26, no. 11, pp. 2131-2144, 2010.

[33] E. Savaş and S. A. Mohiuddine, " $\bar{\lambda}$-statistically convergent double sequences in probabilistic normed spaces," Mathematica Slovaca, vol. 62, no. 1, pp. 99-108, 2012.

[34] E. P. Klement, R. Mesiar, and E. Pap, Triangular Norms, vol. 8 of Trends in Logic, Kluwer Academic Publishers, Dordrecht, The Netherlands, 2000.

[35] A. N. Šerstnev, "Random normed spaces: problems of completeness," Kazanskij Gosudarstvennyj Universitet Imeni V.I. Ul'janova-Lenina. Učenye Zapiski, vol. 122, no. 4, pp. 3-20, 1962.

[36] K. Menger, "Statistical metrics," Proceedings of the National Academy of Sciences of the United States of America, vol. 28, pp. 535-537, 1942.

[37] C. Alsina, B. Schweizer, and A. Sklar, "On the definition of a probabilistic normed space," Aequationes Mathematicae, vol. 46, no. 1-2, pp. 91-98, 1993.

[38] B. Schweizer and A. Sklar, "Statistical metric spaces", Pacific Journal of Mathematics, vol. 10, pp. 313-334, 1960.

[39] B. Schweizer and A. Sklar, ProbabilisticMetric Spaces, Elsevier, New York, NY, USA, 1983.

[40] B. Schweizer and A. Sklar, Probabilistic Metric Spaces, Dover Publication, Mineola, NY, USA, 2nd edition, 2005. 


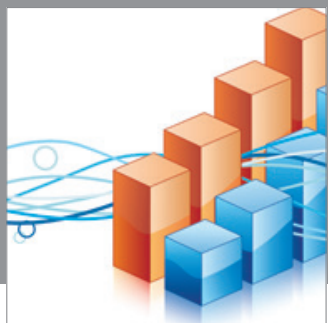

Advances in

Operations Research

mansans

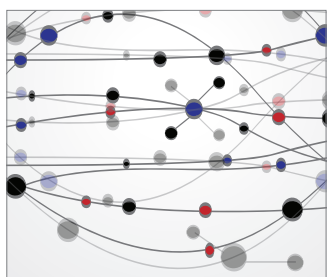

The Scientific World Journal
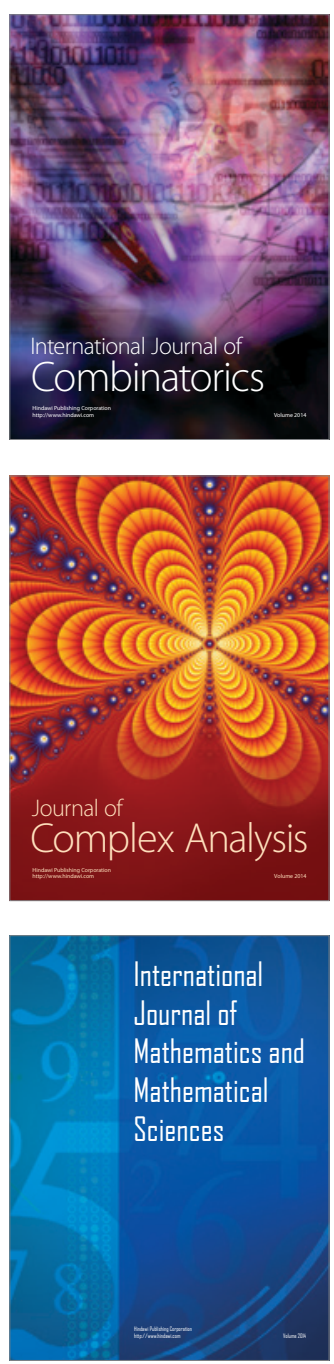
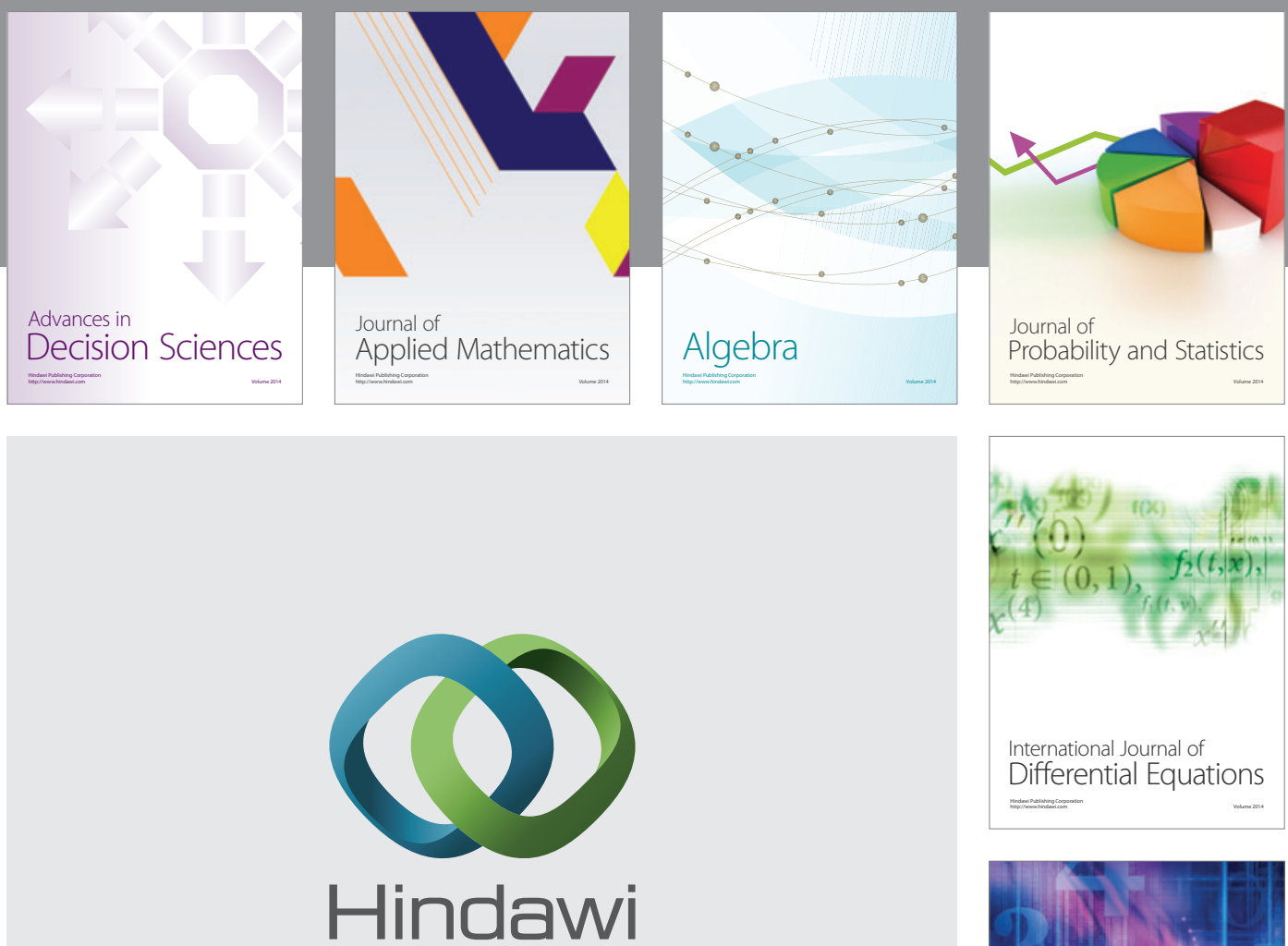

Submit your manuscripts at http://www.hindawi.com
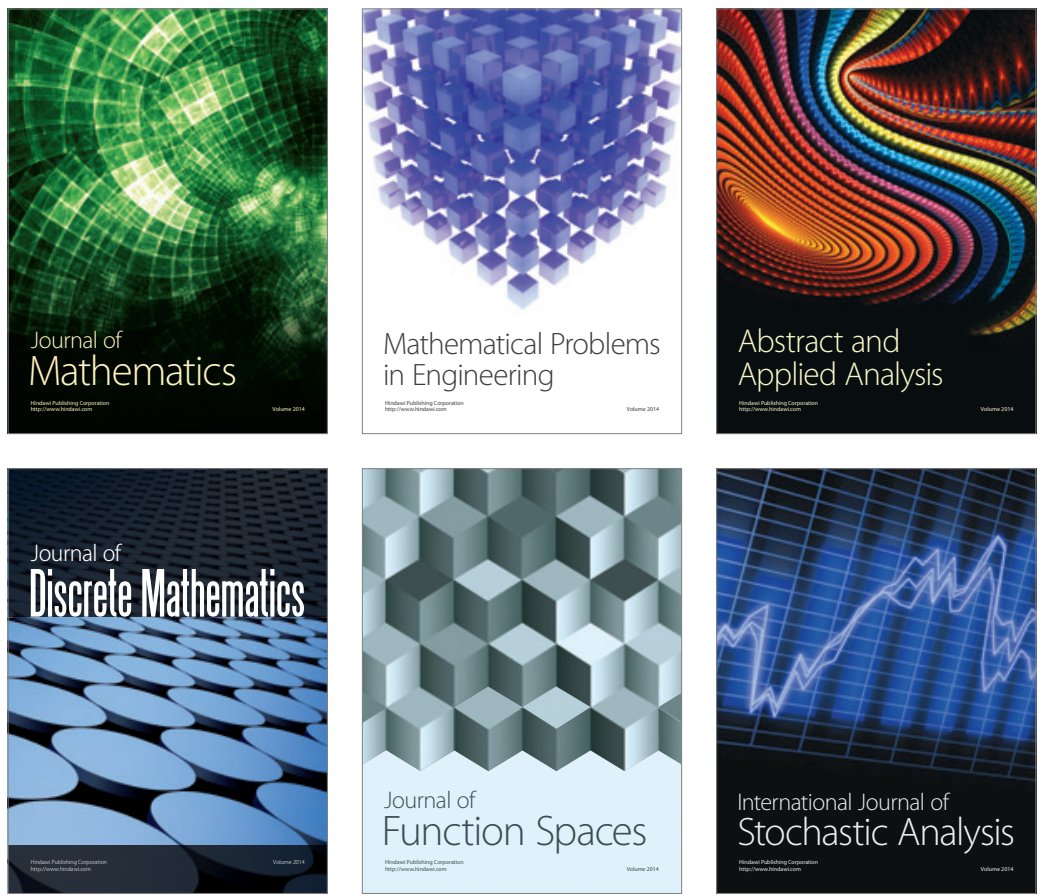

Journal of

Function Spaces

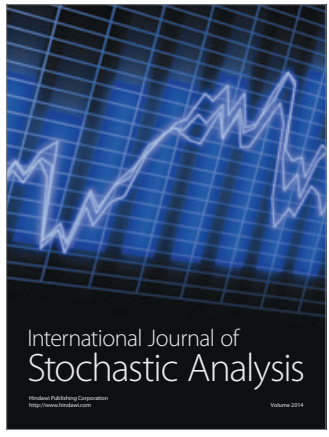

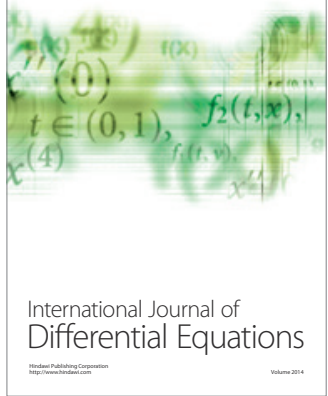
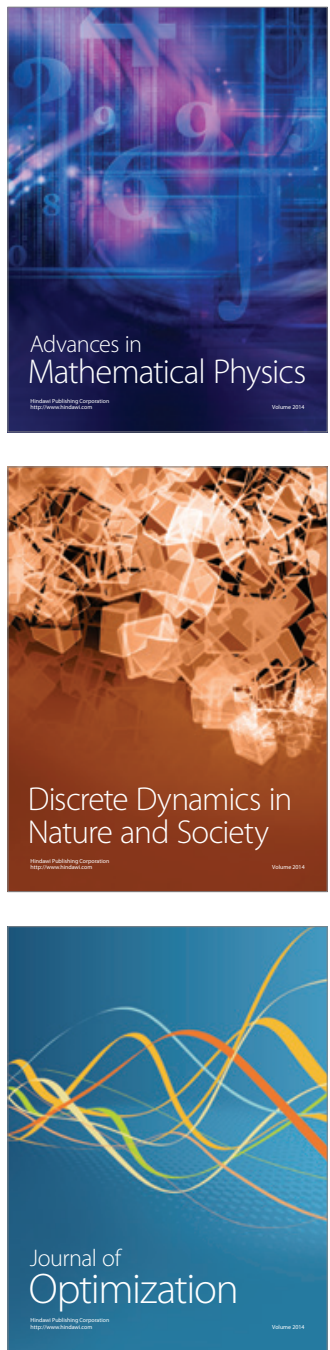